\title{
Adhesional Friction Theory of Micromechanical Surface Contact
}

\author{
Biswajit Bera \\ Department of Mechanical Engineering National Institute of Technology Durgapur, India
}

\begin{abstract}
When two rough surfaces come in contact, tip of asperities would adhere and produces resistance as friction during sliding. First Bowden and Tabor has developed adhesional friction theory based on concept of cold welding of asperity tip through plastic deformation and flow. But this simple theory could not explain for adhesional friction of lightly loaded, clean and smooth hard metallic surface contact (Like MEMS) where asperities deform elastically. In this regard, an alternative adhesional friction theory is developed based on concept of cold welding of asperity through intermolecular adhesion at the area of contact considering JKR and SB adhesion theory of elastic solid sphere.
\end{abstract}

Keywords: Surface contact, Adhesion, Adhesional loading force, Adhesional friction force, Generalized friction law

\section{INTRODUCTION}

Friction could be defined as the force of resistance that occurs when one body moves tangentially over another body. In the 15th century da Vinci discovered a law about dry sliding friction, which was rediscovered by Amontons about 200 years later. Friction laws were stated by French engineer Guillaume Amontons [1] in 1699 from the conclusion of his experimental work which are given as follows:

a) The friction force linearly proportional to the normal load between the two bodies in contact.

b) The friction force is independent of the apparent area of contact between the two bodies.

Thereafter, Bowden and Tabor [2] have first introduced adhesion concept of asperities for rough metallic surface contact. Their simple adhesion theory is classic in the sense that coefficient of friction has represented in term of stress ratio $(\mathrm{S} / \mathrm{H})$ from well known force ratio (friction law) by microscopic investigation of real area of contact. Adhesive bond of asperity junction is explained as cold welding through plastic deformation and flow. However, asperity may deform either elastically or plastically there would be always adhesion and friction in asperity junction. And this simple theory is not able to answer why coefficient of static adhesional friction for lightly loaded, clean and smooth hard metallic surface contact is very high, even greater than 1. Thereafter, Bowden and Tabor revisited their theory and developed modified adhesion theory of friction. It states that if there is application of tangential force on normally preloaded static surface contact, there would be junction growth of the asperity contact area which increases high static adhesional friction. Though this is satisfactorily accepted still now, but it violets well known Mindlin's theory of static friction. Mindlin mentioned that if tangential load is applied on a normally preloaded spherical contact (like asperity contact) there would be tangential deflection under full stick condition with out any junction growth of contact area. Another, important matter is why coefficient of friction for adhesive hard metallic surface contact would be greater than 1 . So, in this regard, there is need of new concept of study for generalized definition of static adhesional friction.

In this study it is considered that asperities should deform elastically and they would cold weld due to intermolecular adhesion at deformed contact zone. And this is investigated in lightly loaded, clean and smooth MEMS surface contact considering adhesion theory of elastic solid sphere. However, there are two type of adhesion theory of elastic solid; one is JKR adhesion model [3] which considers adhesion force within contact area of elastic solid sphere and another is DMT adhesion model [4] which considers adhesive force out side of contact area of elastic solid sphere. First of all, Chang et al. [5] have developed multiasperity adhesion model of metallic rough surface contact based on DMT adhesion model. Thereafter, Roychowdhury and Gosh [6] have considered JKR adhesion model for study of adhesive rough surface contact. Both the two cases, found that external load is evaluated for rough metallic surface contact in presence of adhesion. "Here, author has emphasized on fundamental physics that intermolecular adhesive force within deformed contact zone of asperity produced by external force is responsible for development of adhesional friction resistance at real area of surface contact and so external force along with adhesive force have to be considered for finding coefficient of friction." Actually, Johnson et al. [3] has extended Hertz model considering adhesion of solid elastic sphere based on energy method. Thereafter, on the basis of same method, Savkoor and Briggs [7] has extended JKR adhesion model and developed adhesional friction model of elastic solid sphere under combined normal and tangential loading considering Mindlin's tangential shift. The SB adhesional friction model is considered for finding adhesional friction of clean and smooth MEMS surface contact. 


\section{THEORETICAL FORMULATION}

2.1 Single asperity contact

\subsubsection{Single asperity real area of contact}

JKR adhesion theory has modified Hertz theory of spherical contact. It predicts a contact radius at light loads greater than the calculated Hertz radius. As asperity tip is considered spherical, the adhesion model of single asperity contact could be extended to multiasperity of rough surface contact. So, real contact area of single asperity is

$$
\mathrm{A}_{\mathrm{a}}=\pi\left[\frac{\mathrm{R}}{\mathrm{K}}\left(\mathrm{F}_{0}+3 \pi \gamma \mathrm{R}+\sqrt{6 \pi \gamma \mathrm{RF}_{0}+(3 \pi \gamma \mathrm{R})^{2}}\right)\right]^{\frac{2}{3}}
$$

Substituting $F_{0}=\frac{K(R \delta)^{1.5}}{R}$, we get

$$
\mathrm{A}_{\mathrm{a}}=\pi\left[\mathrm{R}^{1.5} \delta^{1.5}+\frac{3 \pi \gamma \mathrm{R}^{2}}{\mathrm{~K}}+\sqrt{\frac{6 \pi \gamma \mathrm{R}^{3.5} \delta^{1.5}}{\mathrm{~K}}+\frac{9 \pi^{2} \gamma^{2} \mathrm{R}^{4}}{\mathrm{~K}^{2}}}\right]^{\frac{2}{3}}
$$

\subsubsection{Single asperity adhesional loading force}

According to JKR model, the expression of adhesive force for each asperity contact is

$$
\begin{aligned}
& \quad \mathrm{F}_{\mathrm{a}}=\mathrm{F}_{0}+3 \pi \gamma \mathrm{R}+\sqrt{6 \pi \gamma \mathrm{RF}_{0}+(3 \pi \gamma \mathrm{R})^{2}} \\
& =\mathrm{KR}^{0.5} \delta^{1.5}+3 \pi \gamma \mathrm{R}+\sqrt{6 \pi \gamma \mathrm{KR}^{1.5} \delta^{1.5}+(3 \pi \gamma \mathrm{R})^{2}} \\
& \text { where } \frac{1}{\mathrm{~K}}=\frac{3}{4}\left(\frac{1-v_{1}^{2}}{\mathrm{E}_{1}}+\frac{1-v_{2}^{2}}{\mathrm{E}_{2}}\right)
\end{aligned}
$$

and $E_{1}, E_{2}, v_{1}$ and $v_{2}$ are Young's modulus and poisson's ratios of the contacting surfaces respectively, where surface energy of both surfaces, $\gamma=\gamma_{1}+\gamma_{2}-\gamma_{12}$

\subsubsection{Single asperity adhesional friction force}

Savkoor and Briggs theory has found adhesional friction of spherical contact under tangential loading. As asperity tip is considered spherical, the adhesion model of single asperity contact could be extended to multiasperity of rough surface. According to Savkoor and Briggs model, the expression of adhesional friction force for each asperity contact is

$$
\begin{aligned}
\mathrm{T}_{\mathrm{a}} & =\frac{4}{\sqrt{(\mathrm{K} / \mathrm{G}})} \sqrt{\left(2 \pi \gamma \mathrm{RF}_{0}+3(\pi \gamma \mathrm{R})^{2}\right)} \\
= & \frac{4}{\sqrt{(\mathrm{K} / \mathrm{G}}} \sqrt{\left(2 \pi \gamma \mathrm{KR}^{1.5} \delta^{1.5}+3(\pi \gamma \mathrm{R})^{2}\right)}
\end{aligned}
$$

where $\frac{1}{\mathrm{G}}=\frac{2-v_{1}}{\mathrm{G}_{1}}+\frac{1-v_{2}}{\mathrm{G}_{2}}$

\subsection{Multiasperity contact}

First of all, Greenwood and Williamson [8] developed statistical multyasperity contact model of rough surface under very low loading condition and it was assumed that asperities are deformed elastically according Hertz theory. Same model is modified here in adhesive rough surface contact and it is based on following assumptions:

i. The rough surface is isotropic.

ii. Asperities are spherical near their summits.

iii. All asperity summits have the same radius $\mathrm{R}$ but their heights vary randomly followed by Gaussian distribution.

iv. Asperities are far apart and there is no interaction between them

v. Asperities are deform elastically and adhesive bonded according to JKR adhesion theory

vi. There is no bulk deformation. Only, the asperities deform during contact. 


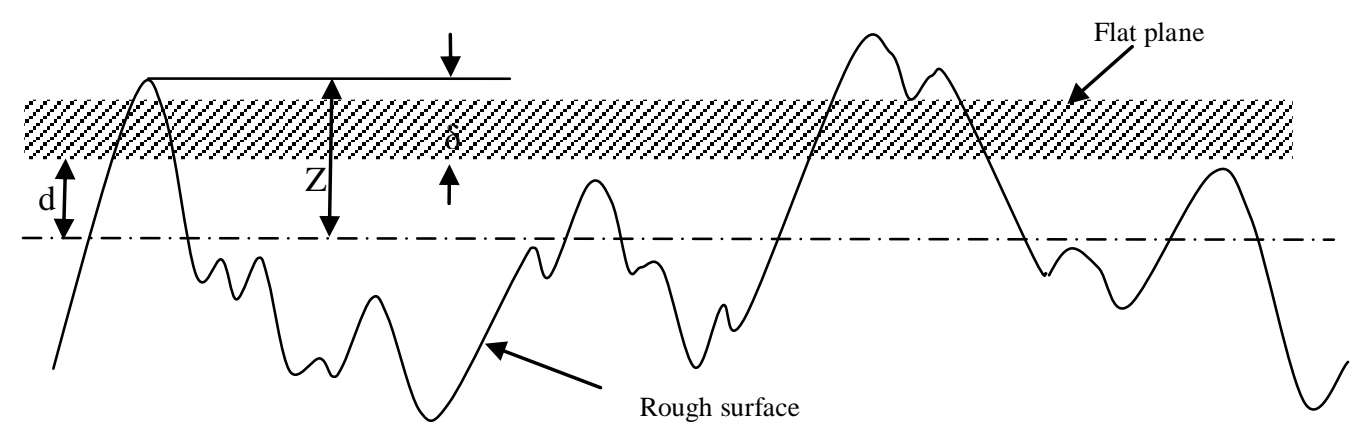

ĕI

Multiasperity contact of adhesive rough surface has shown in Fig.1. According to, GW model, two rough surface contact could be considered equivalently, contact between rough surface and smooth rigid surface. Let $\mathrm{z}$ and $\mathrm{d}$ represents the asperity height and separation of the surfaces respectively, measured from the reference plane defined by the mean of the asperity height. $\delta$ denotes deformation of asperity by flat surface. Number of asperity contact is

$\mathrm{N}_{\mathrm{c}}=\mathrm{N} \int_{\mathrm{d}}^{\infty} \phi(\mathrm{z}) \mathrm{dz}$

where $N$ is total number of asperity and $\phi(z)$ is the Gaussian asperity height distribution function.

\subsubsection{Multiasperity real area of contact}

So, from eq ${ }^{\mathrm{n}}(1)$ and (4), total real area of contact for multiasperity contact is

$$
\begin{aligned}
\mathrm{A}= & \mathrm{N} \int_{\mathrm{d}}^{\infty} \mathrm{A}_{\mathrm{a}} \phi(\mathrm{z}) \mathrm{dz} \\
& =\mathrm{N} \int_{\mathrm{d}}^{\infty} \pi\left[\mathrm{R}^{1.5} \delta^{1.5}+\frac{3 \pi \gamma \mathrm{R}^{2}}{\mathrm{~K}}+\sqrt{\frac{6 \pi \gamma \mathrm{R}^{3.5} \delta^{1.5}}{\mathrm{~K}}}+\frac{9 \pi^{2} \gamma^{2} \mathrm{R}^{4}}{\mathrm{~K}^{2}}\right]^{\frac{2}{3}} \phi(\mathrm{z}) \mathrm{dz}
\end{aligned}
$$

Dividing both side by apparent area of contact $A_{n}$

$$
\begin{aligned}
A^{*} & =\int_{0}^{\infty}\left[\pi^{1.5}(\eta \mathrm{R} \sigma)^{1.5} \Delta^{1.5}+3 \pi^{2.5}(\eta \mathrm{R} \sigma)^{1.5}\left(\frac{\gamma}{\mathrm{K} \sigma}\right)\left(\frac{\mathrm{R}}{\sigma}\right)^{0.5}+\sqrt{6 \pi^{4}(\eta \mathrm{R} \sigma)^{3}\left(\frac{\gamma}{\mathrm{K} \sigma}\right)\left(\frac{\mathrm{R}}{\sigma}\right)^{0.5} \Delta^{1.5}+9 \pi^{5}(\eta \mathrm{R} \sigma)^{3}\left(\frac{\gamma}{\mathrm{K} \sigma}\right)^{2}\left(\frac{\mathrm{R}}{\sigma}\right)}\right] \phi(\Delta) \mathrm{d} \Delta \\
& =\int_{0}^{\infty}\left[\pi^{1.5} \mathrm{~A}_{0}^{1.5} \Delta^{1.5}+3 \pi^{2.5} \mathrm{~A}_{0}^{1.5} \mathrm{~B}_{0} \mathrm{R}_{0}^{0.5}+\sqrt{6 \pi^{4} \mathrm{~A}_{0}^{3} \mathrm{~B}_{0} \mathrm{R}_{0}^{0.5} \Delta^{1.5}+9 \pi^{5} \mathrm{~A}_{0}^{3} \mathrm{~B}_{0}^{2} \mathrm{R}_{0}}\right] \frac{1}{\sqrt{2 \pi}} \exp \left[-\frac{(\mathrm{h}+\Delta)^{2}}{2}\right] \mathrm{d} \Delta
\end{aligned}
$$

\subsubsection{Multiasperity adhesional loading force}

So, , from eq ${ }^{\text {n }}$ (2) and (4), total adhesional loading force for multiasperity contact is $\mathrm{F}=\mathrm{N}_{\mathrm{c}} \mathrm{F}_{\mathrm{a}}$

$=\mathrm{N} \int_{\mathrm{d}}^{\infty}\left[\mathrm{KR}^{0.5} \delta^{1.5}+3 \pi \gamma \mathrm{R}+\sqrt{6 \pi \gamma \mathrm{KR}^{1.5} \delta^{1.5}+(3 \pi \gamma \mathrm{R})^{2}}\right] \phi(\mathrm{z}) \mathrm{dz}$

Dividing both side by $\mathrm{A}_{\mathrm{n}} \mathrm{K}$

$$
\begin{aligned}
\mathrm{F}^{*} & =\int_{0}^{\infty}\left[(\eta \mathrm{R} \sigma)\left(\frac{\mathrm{R}}{\sigma}\right)^{-0.5}+3 \pi(\eta \mathrm{R} \sigma)\left(\frac{\gamma}{\mathrm{K} \sigma}\right)+\sqrt{6 \pi(\eta \mathrm{R} \sigma)^{2}\left(\frac{\gamma}{\mathrm{K} \sigma}\right)\left(\frac{\mathrm{R}}{\sigma}\right)^{-0.5} \Delta^{1.5}+9 \pi^{2}(\eta \mathrm{R} \sigma)^{2}\left(\frac{\gamma}{\mathrm{K} \sigma}\right)^{2}}\right] \phi(\Delta) \mathrm{d} \Delta \\
& =\int_{0}^{\infty}\left[\mathrm{A}_{0} \mathrm{R}_{0}^{-0.5}+3 \pi \mathrm{A}_{0} \mathrm{~B}_{0}+\sqrt{6 \pi \mathrm{A}_{0}^{2} \mathrm{~B}_{0} \mathrm{R}_{0}^{-0.5} \Delta^{1.5}+9 \pi^{2} \mathrm{~A}_{0}^{2} \mathrm{~B}_{0}^{2}}\right] \frac{1}{\sqrt{2 \pi}} \exp \left[-\frac{(\mathrm{h}+\Delta)^{2}}{2}\right] \mathrm{d} \Delta
\end{aligned}
$$

where dimensionless surface roughness parameter, $A_{0}=\eta R \sigma$ and dimensionless surface energy parameter, $\mathrm{B}_{0}=\frac{\gamma}{\mathrm{K} \sigma}$

\subsubsection{Multiasperity adhesional friction force}

So, , from eq ${ }^{\mathrm{n}}$ (3) and (4), total adhesional friction force for multiasperity contact is $\mathrm{T}=\mathrm{N}_{\mathrm{c}} \mathrm{T}_{\mathrm{a}}$ 
$=\mathrm{N} \int_{\mathrm{d}}^{\infty}\left[\frac{4}{\sqrt{(\mathrm{K} / \mathrm{G})}} \sqrt{\left(2 \pi \gamma \mathrm{KR}^{1.5} \delta^{1.5}+3(\pi \gamma \mathrm{R})^{2}\right)}\right] \phi(\mathrm{z}) \mathrm{dz}$

Dividing both side by $A_{n} K$

$$
\begin{aligned}
\mathrm{T}^{*} & =\int_{0}^{\infty}\left[\frac{4}{\sqrt{(\mathrm{K} / \mathrm{G})}} \sqrt{2 \pi(\eta \mathrm{R} \sigma)^{2}\left(\frac{\gamma}{\mathrm{K} \sigma}\right)\left(\frac{\mathrm{R}}{\sigma}\right)^{-0.5} \Delta^{1.5}+3 \pi^{2}(\eta \mathrm{R} \sigma)^{2}\left(\frac{\gamma}{\mathrm{K} \sigma}\right)^{2}}\right] \phi(\Delta) \mathrm{d} \Delta \\
& =\int_{0}^{\infty}\left[\frac{4}{\sqrt{(\mathrm{K} / \mathrm{G})}} \sqrt{2 \pi \mathrm{A}_{0}^{2} \mathrm{~B}_{0} \mathrm{R}_{0}^{-0.5} \Delta^{1.5}+3 \pi^{2} \mathrm{~A}_{0}^{2} \mathrm{~B}_{0}^{2}}\right] \frac{1}{\sqrt{2 \pi}} \exp \left[-\frac{(\mathrm{h}+\Delta)^{2}}{2}\right] \mathrm{d} \Delta
\end{aligned}
$$

\section{RESULTS AND DISCUSSION}

Tayebi and Polycarpou [9] have done extensive study on polysilicon MEMS surfaces and four different MEMS surface pairs. Here, surface roughness, surface energy, and material parameters of the clean and smooth MEMS surfaces are being considered for present study as input data as given in Table 1.The material properties of MEMS surface samples are modulus of elasticity, $\mathrm{K}={ }^{4} /{ }_{3} \mathrm{E}=112 \mathrm{GPa}$, modulus of rigidity, $\mathrm{G}=18.42 \mathrm{GPa}$ hardness, $\mathrm{H}=12.5 \mathrm{GPa}$, and poisions ratio, $v_{1}=v_{2}=0.22$

\begin{tabular}{|c|c|c|c|c|}
\hline $\begin{array}{c}\text { Combined MEMS } \\
\text { Surfaces }\end{array}$ & Rough & Smooth & Intermidiate & Super Smooth \\
\hline $\begin{array}{l}\text { Asperity density } \eta \\
\qquad\left(\mathrm{m}^{-2}\right)\end{array}$ & $14.7 .10^{12}$ & $11.1 .10^{12}$ & $17.10^{12}$ & $26.10^{12}$ \\
\hline Asperity radius $\mathrm{R}(\mathrm{m})$ & $0.116 .10^{-6}$ & $0.45 .10^{-6}$ & $1.7 .10^{-6}$ & $26.10^{-6}$ \\
\hline $\begin{array}{l}\text { Stadandard deviation } \\
\text { of asperity height } \sigma(\mathrm{m})\end{array}$ & $15.8 .10^{-9}$ & $6.8 .10^{-9}$ & $1.4 .10^{-9}$ & $0.42 .10^{-9}$ \\
\hline Surface energy $\gamma(\mathrm{N} / \mathrm{m})$ & 0.5 & 0.5 & 0.5 & 0.5 \\
\hline $\begin{array}{l}\text { Modulus of elasticity K } \\
\qquad\left(\mathrm{N} / \mathrm{m}^{2}\right)\end{array}$ & $112.10^{9}$ & $112.10^{9}$ & $112.10^{9}$ & $112.10^{9}$ \\
\hline $\begin{array}{l}\text { Modulus of rigidity } \mathrm{G} \\
\qquad\left(\mathrm{N} / \mathrm{m}^{2}\right)\end{array}$ & $18.42 .10^{9}$ & $18.42 .10^{9}$ & $18.42 .10^{9}$ & $18.42 .10^{9}$ \\
\hline $\begin{array}{c}\text { Roughness parameter } \\
\qquad \mathrm{A}_{0} \\
\end{array}$ & $27.10^{-3}$ & $34.10^{-3}$ & $41.10^{-3}$ & $53.10^{-3}$ \\
\hline $\begin{array}{c}\text { Surface energy } \\
\text { parameter } \mathrm{B}_{0}\end{array}$ & $2.825 .10^{-4}$ & $6.565 .10^{-4}$ & $31.887 .10^{-4}$ & $74.405 .10^{-4}$ \\
\hline $\begin{array}{l}\text { Asperity radius } \\
\text { parameter } \mathrm{R}_{0} \\
\end{array}$ & 7.342 & 66.176 & 1214.285 & 5600.000 \\
\hline
\end{tabular}

Table.1 Input data

Johnson et. al. first mentioned that deformation of spherical contact would be greater than the deformation predicted by Hertzian spherical contact. It is mentioned that only attractive adhesive force acts within Hertzian contact area and it increases deformation of sphere resulting higher contact area. From Fig.2, dimensionless real area of contact increases with decrement of dimensionless mean separation exponentially. It is found that maximum real areas of contact for the all cases of MEMS surfaces increase as smoothness of MEMS surfaces increase. Dimensionless real area of contact for super smooth MEMS surface is very high almost near to the apparent area of contact due to presence of strong attractive adhesive force. On the other hand, real area of contact is very small for the rough MEMS surface contact. 


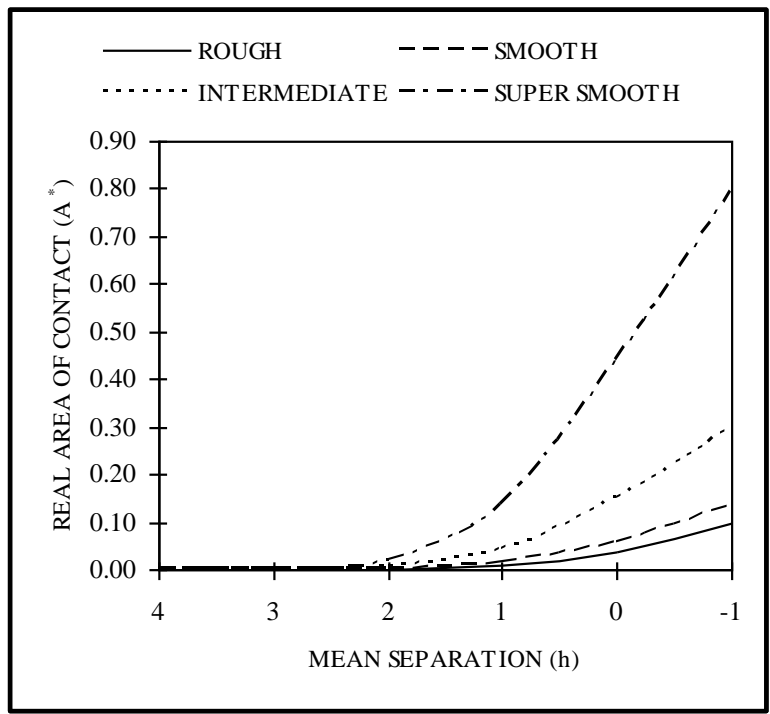

Fig.2 Real area of contact

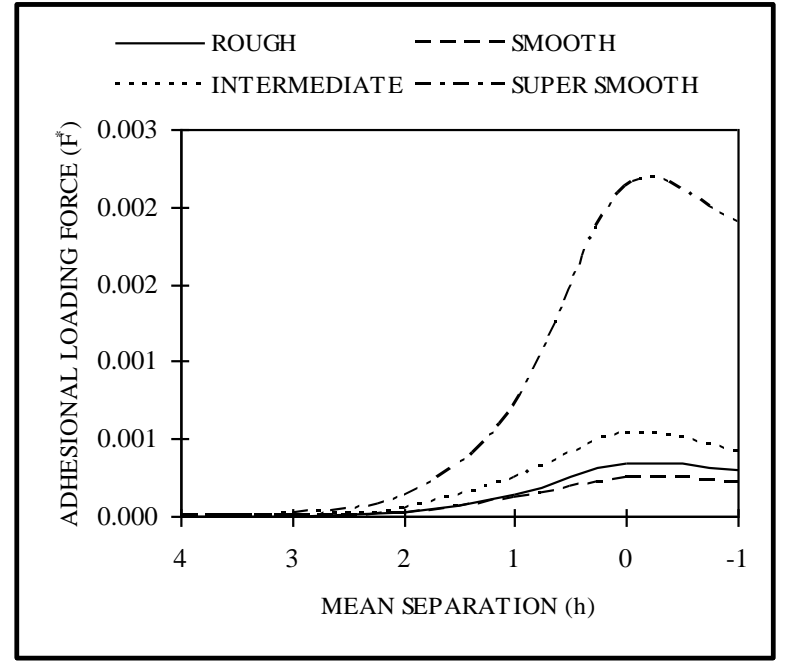

Fig.3 Adhesional loading force

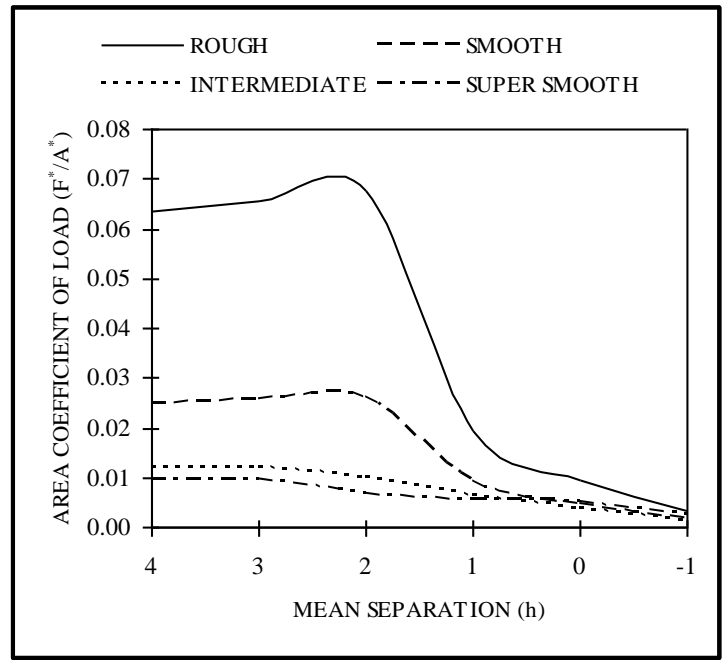

Fig.4 Area coefficient of load

Fig. 3 depicts variation of dimensionless adhesional loading force with dimensionless mean separation. From the loading expression of JKR adhesion model, it is found there is two component of force; one is Hertzian deformation force (i.e. external force) and another is adhesive force. Fig. 3 shows that adhesional loading forces are also increases with decrement of dimensionless mean separation exponentially. In between competing deformation force and adhesive force, as smoothness of MEMS surfaces increases, deformation force decreases but adhesive force increases [10]. So, deformation force and adhesive force are inversely proportional according to consideration of roughness as well as smoothness and there should be a reference MEMS surface where both the force should be minimum such a way that total force should be small. This is happening for smooth MEMS surface. Now, adhesional loading force for rough and intermediate MEMS is much more than that of smooth MEMS surface due to mainly Hertzian deformation force. Similarly, adhesional loading force of super smooth MEMS surface is much more than that of smooth MEMS surface due to mainly high adhesive force Fig.4 shows area coefficient of load verses dimensionless mean separation. This coefficient is considered to understand the relationship in between real area of contact and loading force. Generally, it is assumed that real area of contact and load are linearly proportional as mentioned by Bowden and Tabor considering plastic deformation of asperity. For mean separation smaller than 1, area coefficient for all four cases are comparatively small and are of the slightly descending with same magnitude. However, as mean separation larger than 2, deviation in the coefficient among the four cases becomes increasing prominent and it maintain almost different constant value with mean separation. The separation in between 2 and 1, there is transition of the coefficient from higher different value to lower descending constant value. So, from the discussion, it is found that it do not support the linear relationship in between real area of contact and the loading force. 


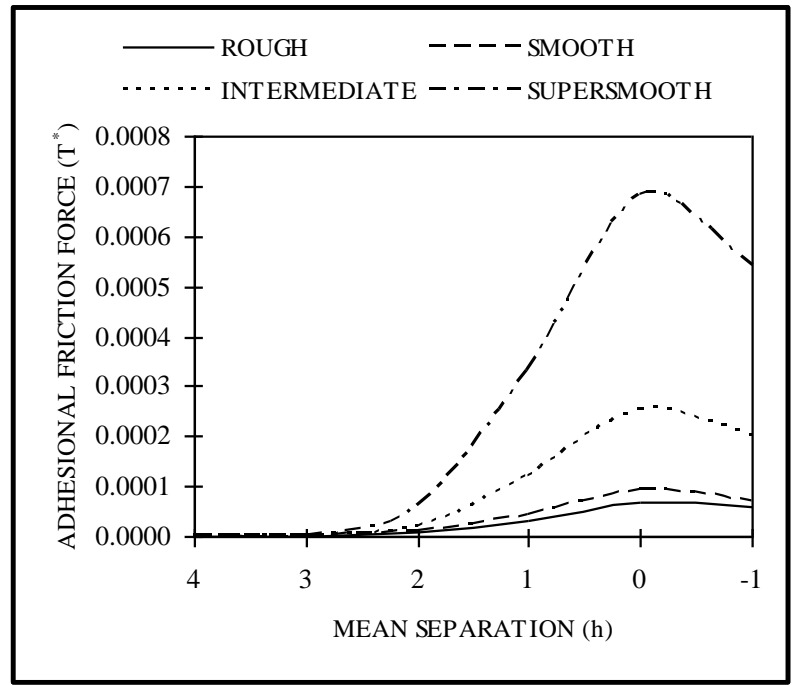

Fig.5 Adhesional friction force

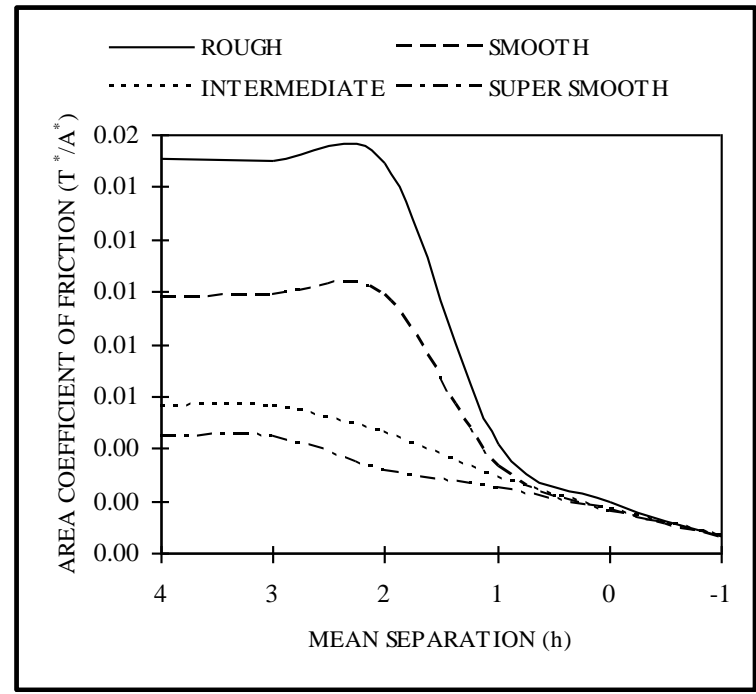

Fig.6 Area coefficient of friction

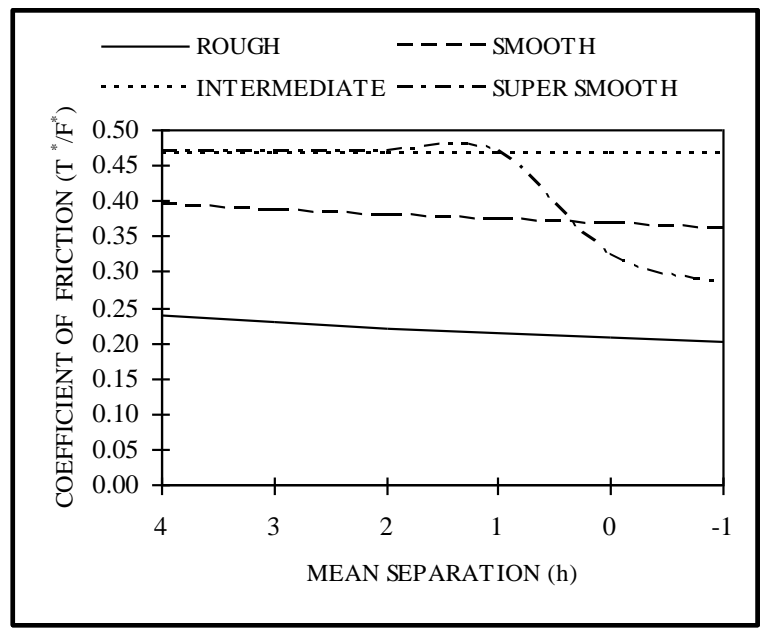

Fig.7 Coefficient of friction

From Fig.5, adhesional friction force increases with decrement of dimensionless mean separation exponentially. It is found that maximum adhesional friction force for the all MEMS surfaces increases as smoothness of MEMS surface increases. Adhesional friction force for super smooth MEMS surface is very high due to presence of strong attractive adhesive force. On the other hand, adhesional friction force is very small for the rough MEMS surface contact. Fig.6 shows area coefficient of friction verses dimensionless mean separation and it follows the similar nature of curve as shown for area coefficient of the loading force. Similarly, It shows non linear relationship in between real area of contact and friction force. Fig. 7 displays variation of coefficient of friction with mean separation. It is found that static coefficient of friction is almost constant except for the super smooth MEMS surface. So, the Amontons law of static friction is validated for adhesive micromechanical surface contact. Only for the supersmooth MEMS surface contact, coefficient of static friction suddenly drops at close contact though nature of curve of adhesional loading force and adhesional friction force for supersmooth MEMS surface is same.

In case of clean and smooth metallic surface contact in vacuum (with out oxidation), it is reported that static coefficient friction is greater than 1 , around 10. It happens due to consideration only external force without adding adhesive force during evaluation of coefficient of friction. If ratio of friction force to contact force ie external force plus adhesive force is evaluated, then coefficient of friction for any adhesive contact would be less than 1 as found in this study. So, there would be no need of Bowden and Tabor's modified adhesion theory of friction for such explanation.

According to Bowden and Tabor's adhesional friction theory, frictional energy dissipation could be explained in term of plastic deformation of asperity. However, in this study, asperity deformation is considered elastic and frictional energy is dissipated through shearing of softer interface and producing adhesive wear particle. 


\section{CONCLUSION}

Finaly, alternative adhesional friction theory could be developed as follows:

- Microscopically, when two rough surfaces come in contact, spherical tip of asperity would deform elastically and adhesive bonding of two asperities is formed due to interatomic adhesion within the deformed contact zone. Subsequent impending sliding of the two rough surfaces produces adhesional friction force at the junction of asperity contact. This intermolecular adhesive bonding could be termed as also cold welding of asperity.

- Real area of contact of rough surface is nonlinearly proportional with adhesional loading and friction force.

- Adhesional friction force is proportionally developed with adhesional loading force due to interatomic adhesive bonding at real area of contact zone. As external load increases, the deformed contact area increases and consequently adhesion within contact zone increases which increases also friction force proportionally. It may be the physical explanation.

- Static coefficient of adhesional friction of the rough surface contact is almost constant and is independent on adhesional loading and friction force.

- Static coefficient of friction increases as smoothness of surface increases.

- New generalized friction law for adhesive contact

Coefficien tof friction $\left(\mu_{\mathrm{s}}\right)=\frac{\text { Friction Force }(\mathrm{T})}{\text { Loading Force }(\mathrm{F})}=\frac{\text { Friction Force }(\mathrm{T})}{\text { External Force }\left(\mathrm{F}_{\text {ext }}\right)+\operatorname{Adhesiveforce}\left(\mathrm{F}_{\text {adh }}\right)}$

Now incase of contaminated or lubricated metallic surface contact where adhesive force is negligible, above generalized friction law would be as usual as friction law for dry friction but it should keep in mind that friction resistance is always developed due to interatomic adhesion within real area of contact however it may be small. Ideally, if it's possible to design a tribosytem where there would be no adhesion within contact zone, the contact would be highly slippery i.e. no friction (like friction for skating over icy surface).

\section{REFERENCES}

[1] G Amontons, Histoire de l'Académie Royale des Sciences avec les Mémoires de Mathématique et de Physique (1699)

[2] F P Bowden and D Tabor, The Friction and Lubrication of Solids (Oxford University Press, New York 1950)

[3] K L Jhonson, K Kendall, and A D Roberts, Surface energy and the contact of elastic solids, Proc. R. Soc. Lond., A 324, 1971, 301-313

[4] B V Derjaguin, V M Muller, and YU P Toporov, Effect of contact deformation on the adhesion of particles, J. Coll. and Inter. Sc., 53, 1975, 314-326

[5] W R Chang, I Etsion, and D B Bogy, Adhesion model for metallic rough surfaces, Journal of Tribology, $110,1988,50-56$

[6] S K Roy Chowdhury and P Ghosh, Adhesion and adhesional friction at the contact between solids, Wear, 174, 1994, 9-19

[7] A R Savkoor and G A D Briggs, The effect of tangential force on the contact of elastic solids in adhesion Proc. R. Soc. Lond., A 356, 1977, 103-114

[8] J A Greenwood, and J B P Williamson, Contact of nominally flat surfaces. Proc. R. Soc. Lond., A 295, 1966, 300-319

[9] N Tayebi and A A Polycarpou, Adhesion and contact modeling and experiments in microelectromechanical systems including roughness effects, Microsyst. Technol., 12, 2006, 854-869

[10] B Bera, Adhesion and adhesional friction of micromechanical surface contact. Journal of Engineering and Applied Sciences, 6, 2011, 104-108 